\title{
Alternative mRNA Splicing in Three Venom Families Underlying a Possible Production of Divergent Venom Proteins of the Habu Snake, Protobothrops flavoviridis
}

\author{
Tomohisa Ogawa ${ }^{1, *}$, Naoko Oda-Ueda ${ }^{2}$, Kanako Hisata ${ }^{3}$, Hitomi Nakamura ${ }^{2}$, \\ Takahito Chijiwa ${ }^{4}$, Shousaku Hattori ${ }^{5}$, Akiko Isomoto ${ }^{6}$, Haruki Yugeta ${ }^{1}$, Shinichi Yamasaki ${ }^{7}$, \\ Yasuyuki Fukumaki ${ }^{6}$, Motonori Ohno ${ }^{4}$, Noriyuki Satoh ${ }^{3}$ and Hiroki Shibata ${ }^{6, *}$ \\ 1 Department of Molecular and Chemical Life Science, Graduate School of Life Sciences, Tohoku University, \\ Sendai, Miyagi 980-8577, Japan; ygthrsc05@gmail.com \\ 2 Department of Pharmaceutical Sciences, Faculty of Pharmaceutical Sciences, Sojo University, Kumamoto \\ 860-0082, Japan; naoko@ph.sojo-u.ac.jp (N.O.-U.); nakamura@ph.sojo-u.ac.jp (H.N.) \\ 3 Marine Genomics Unit, Okinawa Institute of Science and Technology Graduate University, Onna, Okinawa \\ 904-0495, Japan; kanako@oist.jp (K.H.); norisky@oist.jp (N.S.) \\ 4 Department of Applied Life Science, Faculty of Bioscience and Biotechnology, Sojo University, Kumamoto \\ 860-0082, Japan; chijiwa@life.sojo-u.ac.jp (T.C.); mohno218@gmail.com (M.O.) \\ 5 Institute of Medical Science, University of Tokyo, Oshima-gun, Kagoshima 894-1531, Japan; \\ shattori@ims.u-tokyo.ac.jp \\ 6 Division of Genomics, Medical Institute of Bioregulation, Kyushu University, Fukuoka 812-8582, Japan; \\ isomoto@gen.kyushu-u.ac.jp (A.I.); yfukumak@me.com (Y.F.) \\ 7 DNA Sequencing Section, Okinawa Institute of Science and Technology Graduate University, Onna, \\ Okinawa 904-0495, Japan.; shinichi.yamasaki@oist.jp \\ * Correspondence: tomohisa.ogawa.c3@tohoku.ac.jp (T.O.); hshibata@gen.kyushu-u.ac.jp (H.S.); \\ Tel.: +81-22-217-6206 (T.O.); +81-92-642-6168 (H.S.)
}

Received: 31 July 2019; Accepted: 5 October 2019; Published: 9 October 2019

\begin{abstract}
Snake venoms are complex mixtures of toxic proteins encoded by various gene families that function synergistically to incapacitate prey. A huge repertoire of snake venom genes and proteins have been reported, and alternative splicing is suggested to be involved in the production of divergent gene transcripts. However, a genome-wide survey of the transcript repertoire and the extent of alternative splicing still remains to be determined. In this study, the comprehensive analysis of transcriptomes in the venom gland was achieved by using PacBio sequencing. Extensive alternative splicing was observed in three venom protein gene families, metalloproteinase (MP), serine protease (SP), and vascular endothelial growth factors (VEGF). Eleven MP and SP genes and a VEGF gene are expressed as a total of 81,61 , and 8 transcript variants, respectively. In the MP gene family, individual genes are transcribed into different classes of MPs by alternative splicing. We also observed trans-splicing among the clustered SP genes. No other venom genes as well as non-venom counterpart genes exhibited alternative splicing. Our results thus indicate a potential contribution of mRNA alternative and trans-splicing in the production of highly variable transcripts of venom genes in the habu snake.
\end{abstract}

Keywords: venom genes and proteins; metalloproteinase; serine protease; vascular endothelial growth factor; transcriptome variants

Key Contribution: Alternative mRNA splicing in the production of highly variable transcripts of snake venom genes. 


\section{Introduction}

Many snakes have evolved the ability to produce venom, which is a complex mixture of toxic proteins. The toxic proteins contained in the venom are encoded by genes classified into more than ten gene families [1-3]. Protein components of venom are highly variable from species to species. Even within a species, these may vary from population to population and from individual to individual under different physiological and/or environmental conditions. This extensive diversity makes a full characterization of their repertoire difficult. However, recent venomics studies, including high-throughput transcriptomics (RNA-seq), have demonstrated a highly divergent profile of mRNAs encoded by venom genes, and the involvement of alternative splicing is suggested to contribute to transcriptome variety [4-6]. In the era of genome science, the extent of alternative splicing can be examined on a genome-wide scale.

In a previous study, we constructed a draft genome sequence of the habu snake, Protobothrops flavoviridis [7]. An approximately $1.4 \mathrm{Gbp}$ genome assembly, HabAm1, was estimated to contain 25,134 protein-coding genes. A comprehensive annotation of habu genes with the aid of a large transcriptome dataset from 18 different organs has identified a total of 60 venom genes, which are categorized into 18 families [7]. These are metalloproteinase (MP), serine protease (SP), C-type lectin-like proteins (CTLP), phospholipase $\mathrm{A}_{2}$ (PLA2), three-finger toxins (3FTX), aminopeptidases (APaseN), cysteine-rich secretory proteins (CRISP), vespryns/SPla and ryanodine receptor domain proteins (Vespryn), 5'-nucleotidases (5Nase), dipeptidyl peptidases (DDPase), hyaluronidases (Hyal), nerve growth factors or neurotrophins (NGF), vascular endothelial growth factors (VEGF), L-amino acid oxidases (LAAO), phosphodiesterases (PDE), phospholipases B (PLB), bradykinin-potentiating peptides and C-type natriuretic peptides (BNP), and glutaminyl peptide cyclotransferases (GPCase). The first four constitute major families of habu venom proteins [3], including 11 gene copies of $s v M P$ (sv, snake venom) and $s v S P, 10$ of $s v C T L P$, and 9 of $s v P L A 2$. Multiple copies of these gene families were likely formed by multiple rounds of duplications of each of the original venom gene copies [7]. In addition to the accelerated evolution, alternative splicing has been reported as a diversifying mechanism in snake venom proteins such as acetylcholinesterases $[8]$ and svSPs $[9,10]$. However, alternative splicing in venom protein genes has not been genome widely tested. In the present study, we have collected a large quantity of RNA-seq data obtained on a PacBio RSII platform, as well as by Illumina next-generation sequencing (NGS), for the annotation of HabAm1 genes. We describe details of transcripts of venom genes, carefully annotated by using Iso-Seq (isoform sequencing) long reads that span entire transcript isoforms, from the $5^{\prime}$ end to the $3^{\prime}$ polyA-tail. We also addressed the following questions: (1) How many venom genes exhibit alternative mRNA splicing? (2) how many splice variants are produced? and (3) by what mechanisms?

\section{Results}

\subsection{Alternative Splicing Occurs in Three of the 18 Families of Venom Genes}

As indicated, our previous study demonstrated 18 families of 60 venom and 224 non-venom counterpart genes [7]. In the previous study, we also obtained a total 97,405 reads of cDNA collected from the venom gland using PacBio RSII (Iso-Seq) (a total read-length $=179,143,509 \mathrm{bp}$ ). We manually annotated these full-length transcripts corresponding to the gene models previously constructed. As a result, we observed alternative splicing exclusively in three families of venom genes: MPs, SPs, and VEGFs (Figures 1-3). No other families of venom genes, including PLA2s, showed alternative splicing (Figure 4). In other words, each of the transcripts of the remaining 15 venom gene families showed one-to-one correspondence to appropriate venom gene models. svMP and svSP are two of the four major components of habu venom. In contrast, svVEGF is a member of the group in which a single-copy gene is transcribed into one corresponding mRNA. This indicates that (1) alternative splicing contributes to the production of mRNA variants exclusively in venom genes, and (2) alternative splicing occurs only in a few venom gene families, not commonly shared by all venom gene families. 




\section{B SVMP01-HV1 gene}



\section{C svMP02-flavorase gene}



D svMP09-HR1b-related gene

\begin{tabular}{|c|c|c|c|c|c|c|c|c|c|c|c|c|c|c|c|c|c|}
\hline \multirow{2}{*}{$\mathbf{D}$} & & & & \multirow[b]{2}{*}{4} & \multirow[b]{2}{*}{5} & \multirow[b]{2}{*}{6} & \multirow[b]{2}{*}{7} & \multirow[b]{2}{*}{8} & \multirow[b]{2}{*}{9} & \multirow[b]{2}{*}{10} & \multirow[b]{2}{*}{11} & \multirow{2}{*}{\multicolumn{2}{|c|}{$12 \quad 13$}} & \multirow{2}{*}{\multicolumn{2}{|c|}{$\begin{array}{llll}14 & 15 & 16 & 17\end{array}$}} & & \\
\hline & 1 & 2 & 3 & & & & & & & & & & & & & & \\
\hline SVMP09-v1:c917376_f5p9_2037 & M & 口 & 口 & 口 & घ & 口 & n & ! & 口 & 口 & 口 & 口 & 口 & 口 & l & 5 & P-II \\
\hline SVMP09-V2:c228750 f1p2_2017 & M & ㅁ & 믈 & 물 & 믈 & E & ㅁ & 물 & 맘 & 曰 & E & 물 & ㅁ & 口 & - & 7 & P-III \\
\hline svMP09-v3:c415369_f1p4_2324 & $\mathbf{M}$ & - & ㅁ & ㅁ & - & - & = & = & - & - & 口 & - & - & I & 1 & 1 & $P-|| \mid(\Delta D)$ \\
\hline svMP09-v4:c222397_f4p4_2395 & M & : & ㅁ & 믐 & a & E & - & . & - & 口 & - & ㅁ & 口 & 뭄 & 1 & 1 & $\begin{array}{l}\mathrm{B}-\| 11 \\
1-11\end{array}$ \\
\hline SVMP09-v5:c568766_f1p6_4063 & M & - & a & 물 & = & = & ㅁ & 뭄 & [ & 口 & 口 & a & a & 1 & a & 1 & P-II \\
\hline svMP09-v6:c559926_f1p7_4374 & $\mathbf{M}$ & - & ㅁ & ㅁ & घ & 口 & ㅁ & = & 口 & - & - & 口 & r & & $=$ & 1 & P-I \\
\hline
\end{tabular}

\section{E svMP05-HR1a-related gene}



F $\quad$ svMP11-NaMP gene

$$
\text { Exons } \quad \begin{array}{lllllllllllllllll}
1 & 2 & 3 & 4 & 5 & 6 & 7 & 8 & 9 & 10 & 11 & 12 & 13 & 14 & 15 & 16 & 17
\end{array}
$$

SvMP11-v1: habu1_s2862_a10314.12 M $\quad$ a

Figure 1. Cont. 


\begin{tabular}{|c|c|c|c|c|c|c|c|c|c|c|c|c|c|c|c|c|}
\hline \multicolumn{4}{|c|}{ svMP04-jerdonitin-like gene } & \multirow[b]{2}{*}{4} & \multirow[b]{2}{*}{5} & \multirow[b]{2}{*}{6} & \multirow[b]{2}{*}{7} & \multirow[b]{2}{*}{8} & \multirow[b]{2}{*}{9} & \multirow[b]{2}{*}{10} & \multirow[b]{2}{*}{11} & \multirow[b]{2}{*}{13} & \multirow[b]{2}{*}{14} & \multirow[b]{2}{*}{$15(\mathrm{Ex} 17)$} & \multirow[t]{2}{*}{$\begin{array}{l}\text { Number of } \\
\text { IsoSeq reads }\end{array}$} & \multirow[t]{2}{*}{ Types } \\
\hline Exons & 1 & 2 & 3 & & & & & & & & & & & & & \\
\hline SVMP04-v1: c222387 +3p5 2004 & M & - & $\square$ & - & ㅁ & $\square$ & = & m & - & - & - & - & 1 & - & 1 & II \\
\hline SvMP04-v2: c233699_f1p1_1828 & M & ㅁ & E & ㅁ & 므 & = & - & घ & - & ㅁ & 口 & 1 & 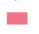 & E & 1 & P-I \\
\hline SVMP04-v3: c230845_f9p5_6405 & $\mathbf{M}$ & ㅁ & - & E & ㅁ & - & = & ㅁ. & - & 물 & 1 & & 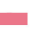 & - & 2 & ו-כ \\
\hline MP04-v4: c82997_f1p2_1888 & м & 口 & E & घ & a & - & - & $\square$ & - & - & 1 & - & घ & $\square$ & 1 & ו-ל \\
\hline MP04-v5: c762_f8p5_1452 & M & 口 & - & - & a & - & - & a & $\mathbf{n}$ & - & 1 & - & - & - & 1 & P-I \\
\hline MP04-v6: c393071_f2p3_3717 & M & ㅁ & E & E & a & m & - & = & - & ㅁ & a & 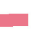 & - & - & 1 & P. \\
\hline
\end{tabular}

H svMP10-elegantin-like gene

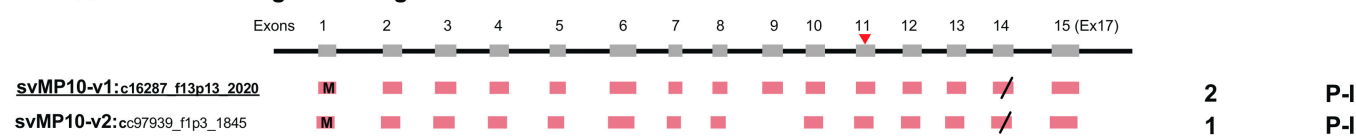

\section{I svMP07-HR2a-related gene}

\begin{tabular}{|c|c|c|c|c|c|c|c|c|c|c|c|c|c|c|c|c|}
\hline Exon & 1 & 2 & 3 & 4 & 5 & 6 & 7 & 8 & 9 & 10 & $11 \quad 12$ & 13 & 14 & $15(\mathrm{E} \times 17)$ & & \\
\hline SVMP07-v1: c226 f26p9 2010 & M & [ & 口 & [ & E & m & 口 & - & - & 口 & 口 & 口 & 1 & - & 6 & \\
\hline svMP07-v2: c412727_f2p12_1989 & M & 口 & ㅁ & [ & E & m & घ & a & ㅁ & 무 & - & - & - & 1 & 2 & \\
\hline svMP07-v3: c552023_f10p36_4003 & M & - & $\mathbf{E}$ & = & - & $=$ & E & - & 뭄 & 무 & 1 & - & - & ש & 3 & \\
\hline svMP07-v4: c230957_f2p12_3969 & M & ㅁ & ש & - & 무 & $=$ & = & = & - & 口 & 1 & ㅁ & - & $=$ & 1 & \\
\hline SvMP07-v5: c222038_f23p8_2094 & M & - & - & - & - & $=$ &  & - & - & ㅁ & $1=$ & - & - & - & 2 & \\
\hline svMP07-v6: c410202_f2p6_4970 & M & 口 & 무 & - & - & $=$ & घ & - & 무 & - & 1 & 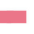 & - & $=$ & 1 & \\
\hline svMP07-v7: c182477_f1p6_4962 & M & 口 & 口 & - & घ & - & घ & a & - & 口 & / & 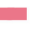 & I & - & 1 & \\
\hline SVMP07-v8: c569924__3p28_4139 & M & 口 & - & 口 & घ & - & r &  & - & 口 & 口 & - & 口 & - & 1 & \\
\hline svMP07-v9: c735029_f6p24_6129 & M & 口 &  & = & ㅁ & $=$ & Y & &  & 무 &  & = & 무 & $=$ & 2 & \\
\hline svMP07-v10: c301942_f1p9_7082 & M & - & - & m & - & = & I & ta & 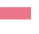 & 口 & &  & - & - & 1 & 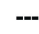 \\
\hline svMP07-v11: c92848_f1p2_1157 & M & - & - & - & - & = & $=7$ & 1 & - & - & ㅁ & $\square$ & - & - & 1 & 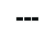 \\
\hline svMP07-v12: c181237_f1p2_4088 & M & ㅁ & - & - & = & - & $\pm \gamma$ & 1 & - & 口 & 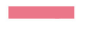 & 물 & - & - & 1 & \\
\hline sVMP07-v13: c164930_22p0_4266 & M & E & - & ㅁ & E & 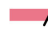 & & $\mathbf{E}$ & 밀 & ㅁ & E & E & - & ㅁ & 1 & \\
\hline
\end{tabular}

\section{J svMP08-flavoridin gene}

SVMP08-v1: c222 f46p26_1997

\section{K sVMP06-H2-protease gene}

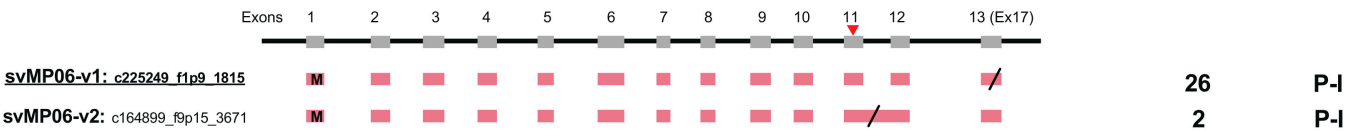

Figure 1. Schematic diagram of structures of metalloproteinase (MP) genes expressed in habu venom glands. svMP03-VMP-III-like (A), svMP01-HV1 (B), svMP02-flavorase (C), svMP09-HR1b-related (D), svMP05-HR1a-relate (E), svMP11-NaMP (F), svMP04-jerdonitin-like (G), svMP10-elegantin-like (H), svMP07-HR2a-related (I), svMP08-flavoviridin (J), and svMP06-H2-protease $(\mathbf{K})$ are shown with validated transcript variants, verified as expressed in the venom gland. Initiation and stop-codons are marked with " $\mathrm{M}$ " and slashes, respectively. Active sites are shown with red arrowheads located in exon 11. The original transcripts predicted by the gene models are underlined. Both svMP08-v1 and v2 are original transcripts, of which amino acid 275 are indicated by $\mathrm{E}(\mathrm{Glu})$ and $\mathrm{K}$ (Lys), respectively. Numbers of transcript variants and the type of metalloproteinases are shown on the right. 

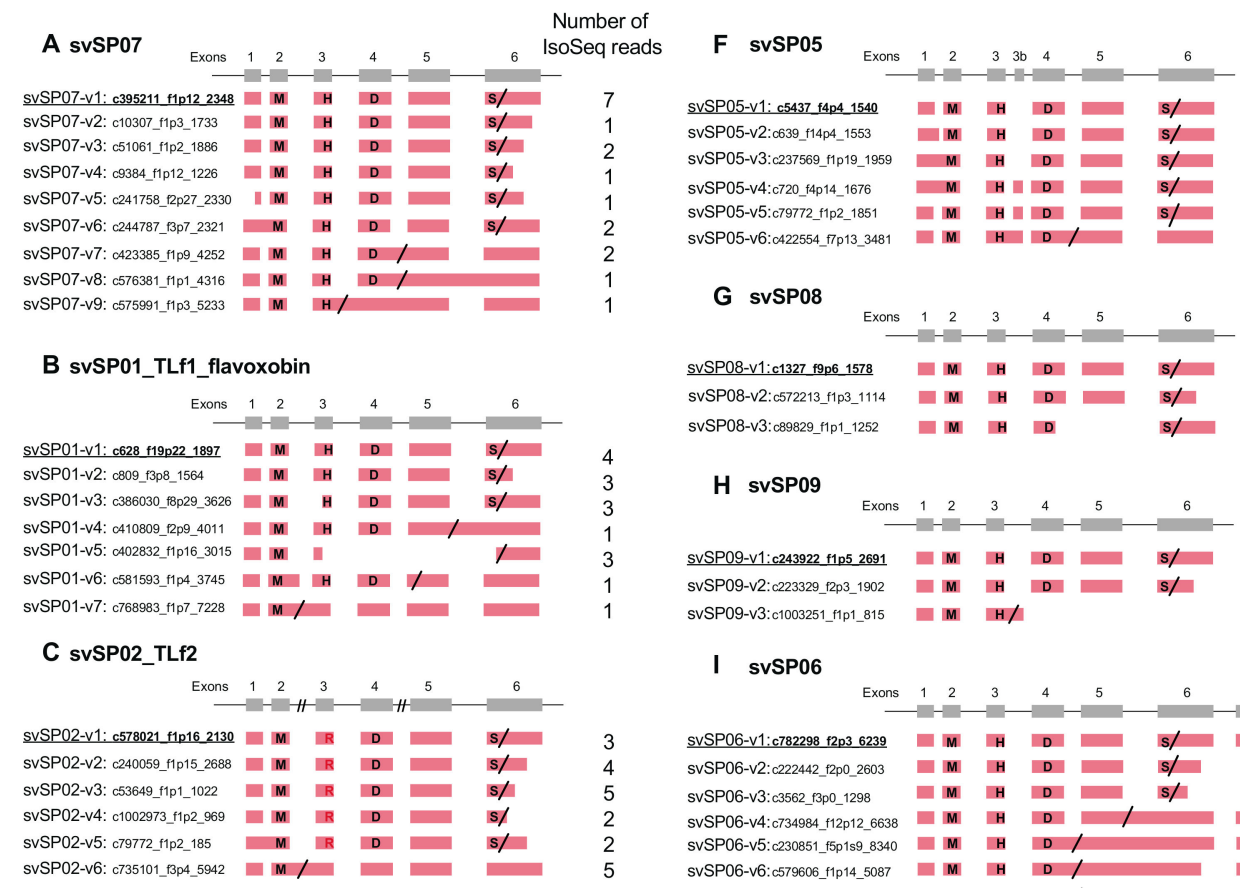

Number of IsoSeq reads
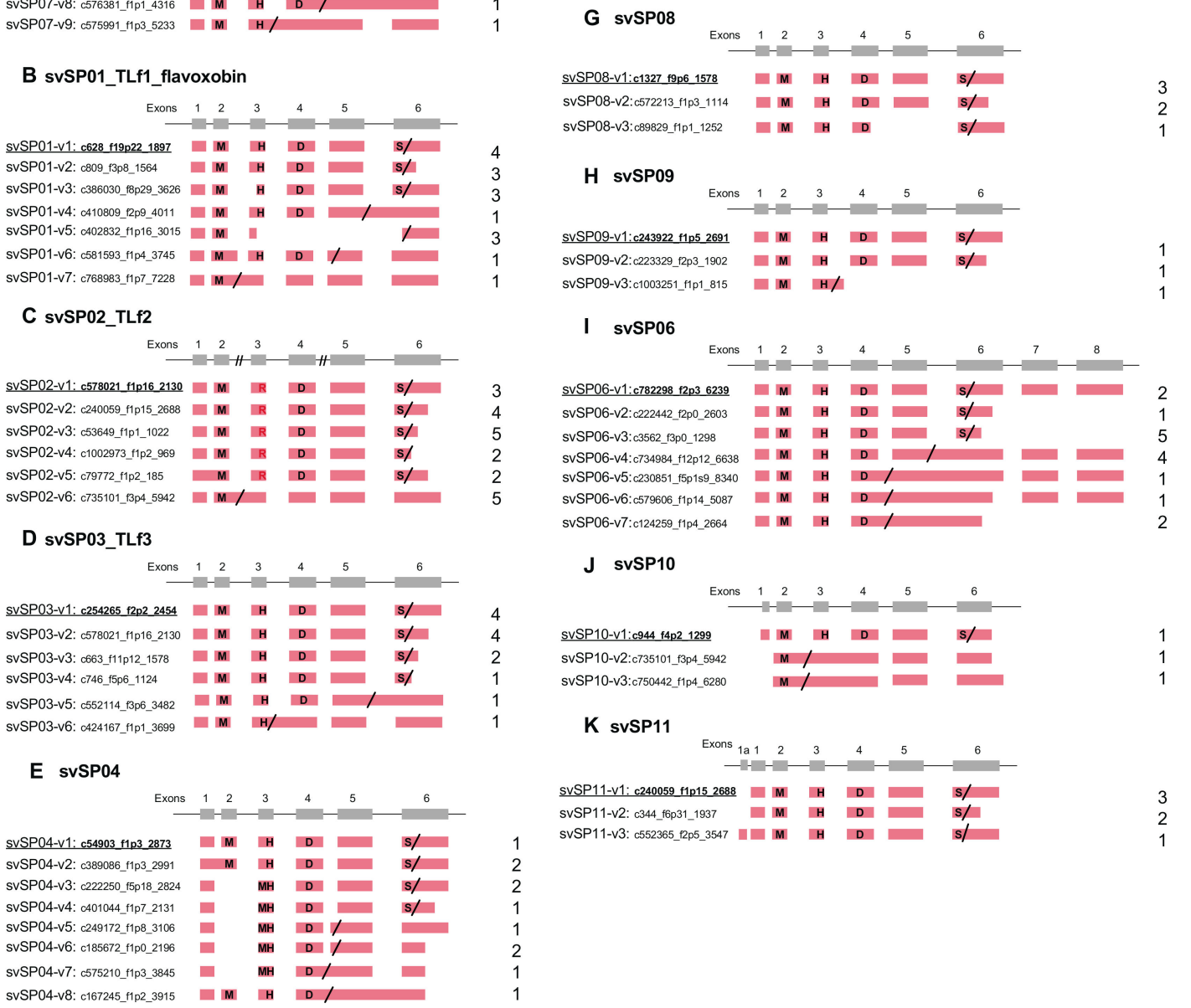

Figure 2. Schematic diagram of structures of serine protease (SP) genes expressed in habu venom glands. svSP07 (A), svSP01-TLf1-flavoxobin (B), svSP02-TLf2 (C), svSP03-TLf3 (D), svSP04 (E), svSP05 (F), svSP08 (G), svSP09 (H), svSP06 (I), svSP10 (J), and svSP11 (K) are shown with validated transcript variants, verified as expressed in the venom gland. Initiation and stop-codons are marked with " $\mathrm{M}^{\prime}$

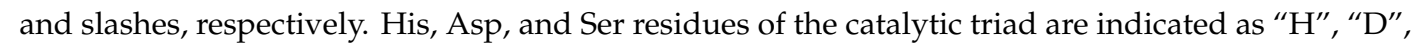
and " $S$ ", respectively. In svSP02-TLf2, the His residue " $\mathrm{H}$ " of the catalytic triad is substituted by Arg (R). Numbers of transcript variants are shown on the right. The original transcripts predicted by the gene models are underlined. 


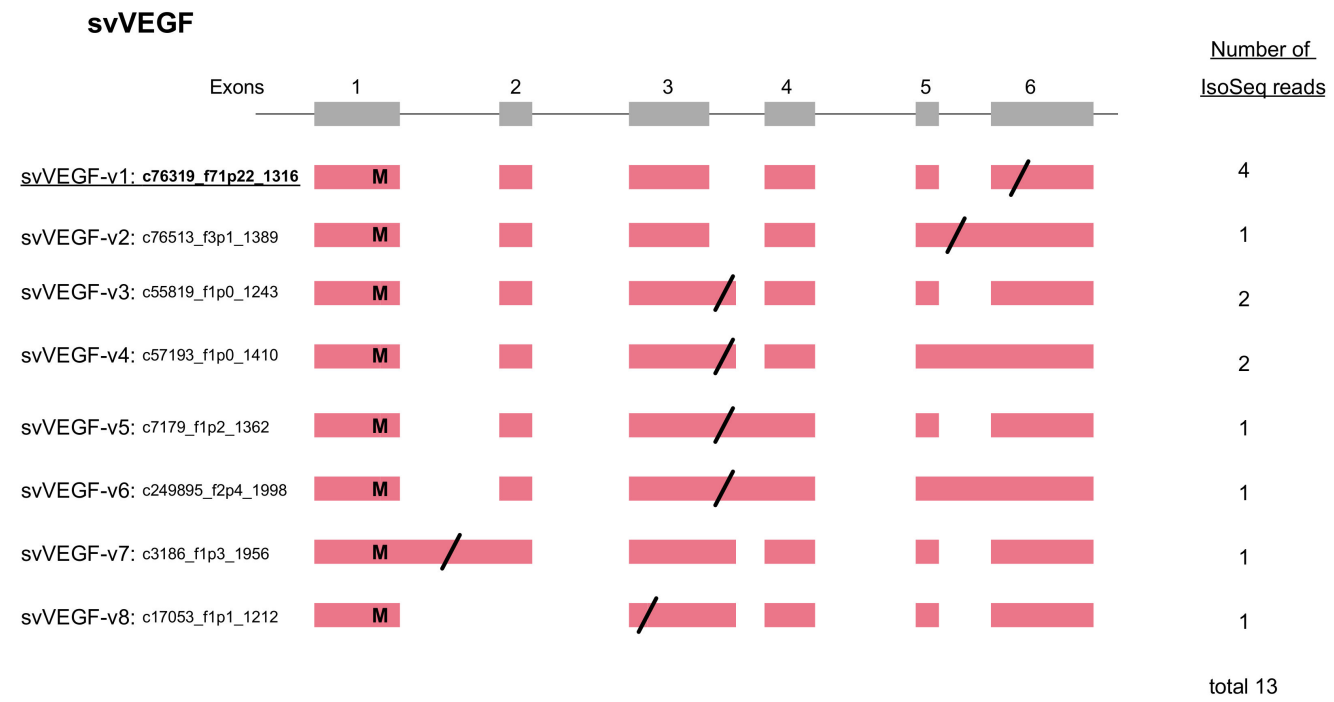

Figure 3. Schematic diagram of structure of vascular endothelial growth factor (VEGF) genes expressed in habu venom glands. Initiation and stop-codons are marked with " $\mathrm{M}$ " and slashes, respectively. Seven transcript variants, svVEGF-v1 to v8, were identified. Numbers of transcript variants are shown on the right. The original transcript predicted by the gene models is underlined.

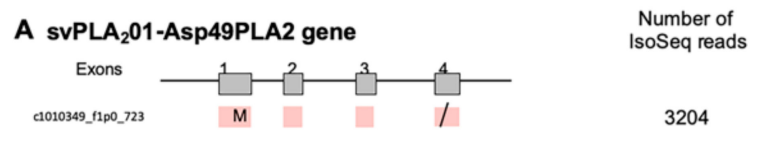

B svPLA 2 02-PLX'/PL-Y/PLA-B genes

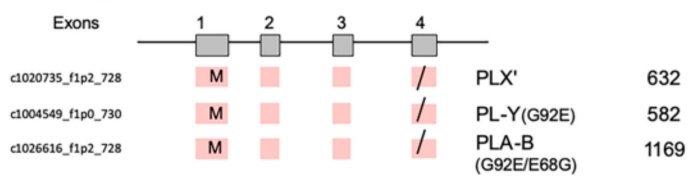

\section{C svPLA $203-P L A-N$ gene}

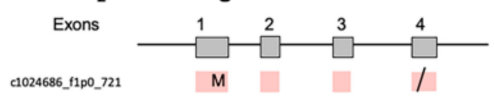

181

\section{D svPLA 2 04/05/06-BPI/BPII/BPIII genes}

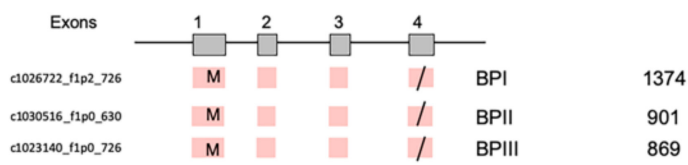

E svPLA 2 07-pgPL1b/PL2b gene

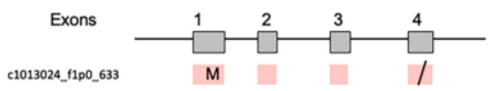

726

F svPLA $208-$ PfPLA6 gene

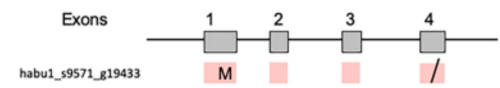

G svPLA 2 09-pgPLA2b gene

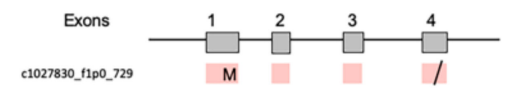

Figure 4. Schematic diagram of structure of phospholipase $\mathrm{A}_{2}$ (PLA2) genes expressed in habu venom glands. svPLA2_01-Asp49PLA2 (A), svPLA2_02-PLX'/PL-Y/PLA-B (B), svPLA2_03-PLA-N (C), svPLA2_04/05/06-BPI/BPII/BPIII (D), svPLA2_07-pgPL1b/PL2b (E), svPLA2_08-PfPLA6 (F), and svPLA2_09-pgPLA2b (G) are shown with validated transcript variants expressed in the venom gland. 
Initiation and stop-codons are marked with " $\mathrm{M}$ " and slashes, respectively. Numbers of transcript variants are shown on the right.

\subsection{Alternative Splicing of 10 svMP Genes Produces $81 \mathrm{mRNA}$ Variants}

MPs are key toxins that cause venom-induced pathogenesis such as hemorrhage, fibrinolysis, and apoptosis. According to their domain architecture, svMPs are classified into four groups (P-I to P-IV) [11] (Figure 5A). P-I MPs possess only the metalloproteinase domains and are largely non-hemorrhagic. P-II MPs contain MP domains and disintegrin domains. P-III MPs contain Cys-rich domains as well as MP and disintegrin domains and exhibit great diversity in structure and function. P-IV MPs harbor lectin-like domains linked by disulfide bonds to the P-III-like structures [11]. Eleven svMP genes have been annotated from the habu gene model, HabAm1 (svMP01 to svMP11) (Figures 1 and 5B; Supplementary Table S1).

In the present study, we obtained 1777 PacBio reads related to the svMPs, however only 1032 PacBio reads out of 1777 can be assigned on the svMP genes, svMP01 to svMP10. Residual 745 PacBio reads for svMPs could not assigned on $s v M P$ genes probably due to their non-perfect match to the known svMPs. This suggests that the additional new svMP genes exist in the habu genome. After removing the short PacBio reads (total 839 reads), 193 full-length transcripts (PacBio reads) that include both start Met codon and polyA signal were used for further analysis.

As shown in Figures 1 and 5B, the svMP genes consist of 13 to 17 exons. Six svMP genes, svMP01-HV1, svMP02-flavorase, svMP03-VMP-III-like, svMP05-HR1a-related, svMP09-HR1b-related and svMP11-NaMP-like consist of 17 exons. Four svMP genes, svMP04-jerdonitin-like, svMP07-HR2a-related, svMP08-flavoridin, and svMP10-elegantin-like consist of 15 exons. Only svMP06-H2-protease consists of 13 exons. Exon 1 encodes a signal sequence, and exons 2 to 6 encode pre-prosequences. Exons 7 to 12 encode MP domains, including a catalytic zinc binding motif, HEXGHNLGXXHD encoded by exon 11 in all svMP genes (Figure 5A). Furthermore, exons 13 and 14 encode the disintegrin domains of P-II and P-III svMPs, and exons 15 and 16 encode the Cys-rich domain of P-III svMPs (Figure 5A). A molecular phylogenetic tree constructed from nucleotide sequences of $P$. flavoviridis svMPs is shown in Figure 5B. The gene structure with 17 exons, as seen in svMP11-NaMP-like, is suggested to be ancestral due to its basal location in the phylogenetic tree (Figure 5B). svMP02-flavorase, svMP01-HV1, svMP03-VMP-III-like, svMP05-HR1a-related, and svMP09-HR1b-related have diverged from this ancestor without any structural changes to exons. On the other hand, exons 15 and 16 corresponding to the Cys-rich domain were deleted in the four genes consisting of 15 exons: svMP04-jerdonitin-like, svMP10-elegantin-like, svMP07-HR2-related, svMP08-flavoridin, and svMP06-H2-protease (Figures 1 and 5B).

In the case of svMP06-H2-protease, exons 13 and 14 corresponding to the disintegrin domain were further deleted, resulting in 13 exons (Figure 5B). An important notion is that the last exon, exon 17 in the original construct, is conserved in all of the "reduced" gene copies consisting of 13 or 15 exons. These gene structures suggest that the ancestral svMP gene copy related to svMP11-NaMP consisting of 17 exons duplicated into multiple copies, and some of the copies were then modified by two-round deletions, the first one involving loss of exons 15 and 16, and the second one resulting in further loss of exons 13 and 14, resulting in 11 svMP gene copies with different numbers of exons (Figure 5B).

All of the 81 full-length mRNAs expressed in the venom gland matched each of the corresponding gene models. We identified multiple kinds of transcripts for each of the MP genes except for svMP11-NaMP, which expressed only one type of the transcript (Figure 1F; Supplementary Table S1). We hereafter call the transcript completely matching the corresponding gene model as "original" represented by svMP03-v1 for svMP03-VMP-III-like supported by six Iso-Seq reads (Figure 1A). Besides the original transcripts, we observed 2, 5, 6, 5, 18, 0, 5, $1,12,16$, and 1 variant transcripts for the other $M P$ genes. (Figure $1 \mathrm{~A}-\mathrm{K}$ ). These variants include transcripts, of which $3^{\prime}$ untranslated regions' ( $3^{\prime}$ UTR) length is the only thing that is different due to having multiple polyA signals AATAAA, and which are able to translate the same protein products. For example, they include svMP03-v1 and svMP03-v2 for svMP03-VMP-III-like, svMP01-v1, svMP01-v2 and svMP01-v3 for svMP01-HV1, svMP02-v1, svMP02-v2 and svMP02-v3 
for svMP02-flavorase, and svMP09-v2 and svMP09-v3 for svMP09-HR1b. We also observed variable positions of the stop-codon in svMP01-HV1-related, svMP02-flavorase-related, svMP09-HR1b-related, svMP05-HR1a-related, svMP04-jerdonitin-like, svMP07-HR2a-related, svMP08-flavoridin, and svMP06-H2-protease (Figure 1B-E,G,I-K). Most of these differences are associated with exon skipping or intron retention followed by frame shifts. For example, three different transcript variants of svMP09-HR1b show variable positions of the stop-codon, svMP09-v4 skipping exon 15 with a new stop-codon in exon 16, svMP09-v6 retaining intron 13 with a new stop-codon in the newly fused exon (exons 13 and 14), and svMP09-v5 retaining intron 14 with a new stop-codon in the newly fused exon (exons 14 and 15) (Figure 1D), resulting in the variants corresponding to P-I and P-II MPs, respectively.

A

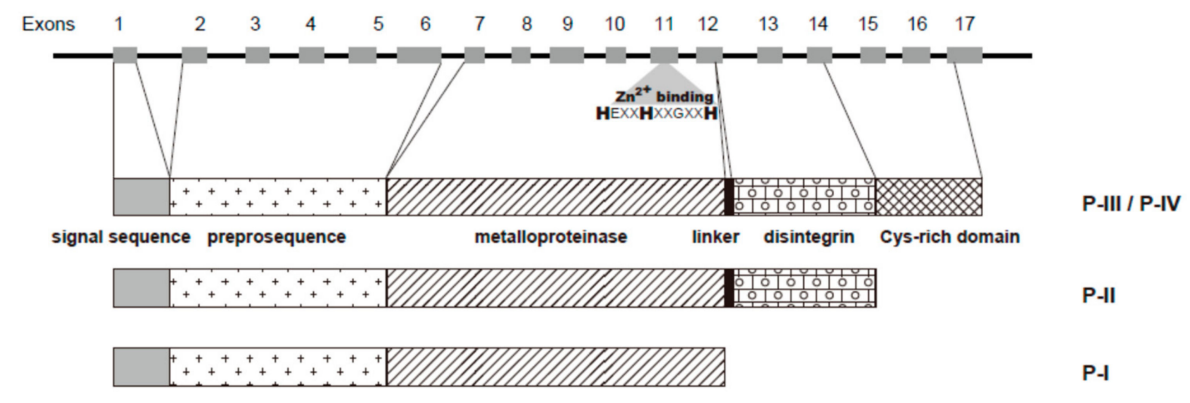

B

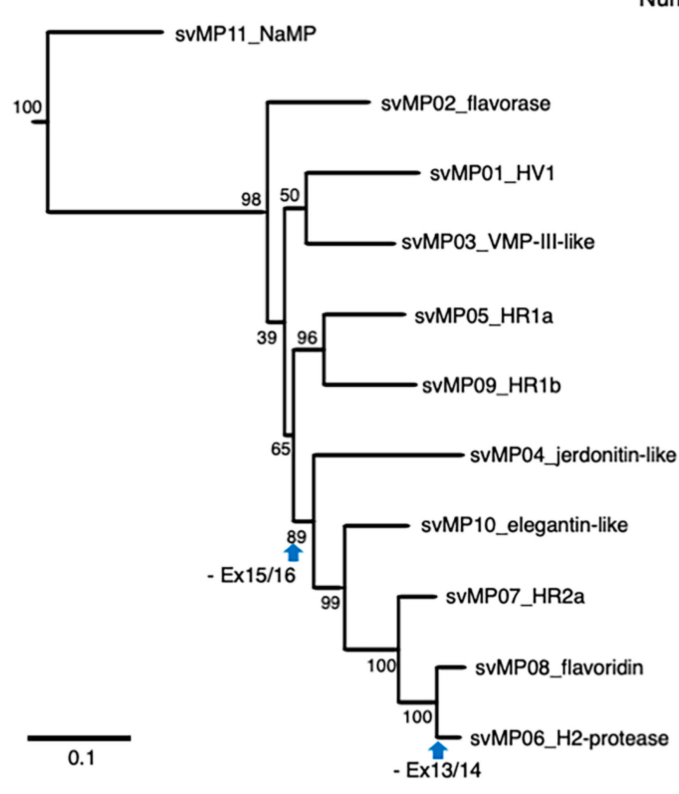

Number of Exons Transcripts

$17 \quad 7$

$17 \quad 6$

$17 \quad 3$

$17 \quad 18$

$17 \quad 6$

$15 \quad 5$

152

$15 \quad 13$

$15 \quad 17$

132

Figure 5. Schematic structures (A) and phylogenetic tree (B) of metalloproteinase genes expressed in habu venom glands. (A) The gene structure is shown with 17 exons. A Zn binding site is also shown in exon 11. Different domains in the three types of MP protein products (P-I, P-II, and P-III/P-IV) are shown as boxes. The active site is also shown by asterisk as " $\mathrm{Zn}^{2+}$ binding" motif, HEXGHNLGXXHD. P-IV contains lectin-like domain linked by disulfide bonds to the P-III structures. (B) Molecular phylogeny of $11 M P$ genes. Three non-venom MPs found in the habu genome, nvMP08, nvMP09, and nvMP11 were used as outgroups for rooting the tree. Bootstrap probabilities of 1000 replications are shown at each node. Deduced timings of gain and loss of exons are shown by blue arrows. Exon structures and numbers of transcript variants are also shown on the right. 
The most extensive variations were noted in svMP05-HR1a-related, showing the original transcript svMP05-v1 supported by 10 Iso-Seq reads and 17 variant transcripts, svMP05-v2 to svMP05-v18, supported by 1 to 4 Iso-Seq reads, respectively (Figure 1E). Unlike other svMP genes, extra sub-exons $(1 \mathrm{~b}, 1 \mathrm{c}, 1 \mathrm{~d}$, and 1e) were observed between the original exon 1 and exon 2 in some transcript variants such as svMP05-v2, The original transcript and 12 variants contained only the original exon 1a, while another six transcript variants exhibited more complicated combinations of exon usage characterized by loss or fusion of certain exons (svMP05-v2, svMP05-v3, svMP05-v4, svMP05-v5, and svMP05-v6 in Figure 1E). Five of the six variants also showed different positions of the start-codon due to different exon usage (svMP05-v3, svMP05-v4, svMP05-v5, and svMP05-v6 in Figure 1E). Six exons, exon 7 to exon 12 encoding the MP domain, including the catalytic Zn finger motif (in exon 11), are conserved in all of the observed transcripts, suggesting that the six exons are essential for enzyme function (Figures 1 and 5A). It is notable that the original transcript of svMP05-HR1a-like encodes P-III MP, while two other types of svMPs are also transcribed from the same gene by alternative splicing. Six transcripts encode P-I type, in which a stop-codon was introduced after exon 12 due to the retention of intron 12 (transcripts svMP05-v12, svMP05-v14, svMP05-v15, and svMP05-v16). Another two transcripts encode P-II-type in which a stop-codon was introduced after exon 14 due to the retention of intron 14 (transcript: svMP05-v9) or shortening of exon 14 (transcript: svMP05-v10).

svMP04-jerdonitin-like, svMP10-elegantin-like, svMP07-HR2a-related, and svMP08-flavoridin are likely to comprise another group of $s v M P$ genes of which the original construct is composed of 15 exons encoding P-II MPs (Figure 1G-J). As observed above for the genes that originally consisted of 17 exons, variable transcripts were produced by exon skipping and intron retention (Figure 1G-J). The most extensive variation in this group was observed in svMP08-flavoridin, which is expressed as 15 variant transcripts and two original ones (Figure 1J). Both svMP08-v1 and svMP08-v2 were original transcripts, of which amino acid 275 is replaced by Glu or Lys. Another transcript, svMP08-v3, carrying fused exons 14 and 15 by retaining intron 14 was a P-II type with a disintegrin domain. However, three variant transcripts, svMP08-v 4 carrying fused exons 13 and 14 by retaining intron 13, svMP08-v5 eliminating exon 14 and svMP08-v6 eliminating exons 14 and 15, were P-I type. Extensive variations were also observed in svMP07-HR2a-related, expressed as 12 variant transcripts, svMP07-v2 to v13 as well as the original transcript (Figure 1I). Although the first five exons, exon 1 to 5 , are shared by all of the nine transcripts, intron retentions are involved in the variant transcripts, svMP07-v3 carrying fused exons 11 and 12 by retaining intron 11, svMP07-v2 carrying short exon 14, c110395_f1p2_2139 carrying extended exon 8, svMP07-v5 carrying extended exon 11 by partial retain of intron 11, svMP07-v13 retaining intron 6, svMP07-v8 retaining intron 7, svMP07-v9 retaining introns 7 and 11, and svMP07-v11 and svMP07-v12 partially retaining intron 7 (Figure 1I). New stop-codons are gained in the retained intron 11 in two transcripts encoding P-I MPs: svMP07-v3 and svMP07-v5, which can be produce the same protein. Lastly, svMP06-H2-protease, consisting of 13 exons, is transcribed into a variant, svMP06-v2 gaining a stop-codon in the retained intron 11 as well as the original construct (Figure 1J).

To confirm the expression level of each transcript variant for svMPs, we analyzed the prevalence and relative abundance of the splice variants in venom gland by HISAT (hierarchical indexing for spliced alignment of transcripts), StringTie, and Ballgown with Illumina RNA-Seq data (Figure 6). The relative abundance of the splice variants for other svMPs were obtained except for svMP04 and svMP09. They could not be obtained due to their gene structures locating across the multi-scaffolds, that is, svMP04 is located on habu1_scaffold415864, habu1_scaffold191139, and habu1_scaffold403873, and svMP09 is located on habu1_scaffold3258, habu1_scaffold399953, habu1_scaffold411246, habu1_scaffold399953, and habu1_scaffold400013. These data suggest the existence of the several splice variants of svMPs in venom gland. For example, in the case for svMP05-HR1a gene, distinct expression of transcript variants for svMP05-v1 to v18 was detected by Illumina RNA-seq except for svMP05-v6, svMP05-v7, svMP05-v8, svMP05-v10, svMP05-v12, and svMP05-v16. These differences of expression profiles between Illumina RNA-seq and PacBio reads may be individual specificities. Interestingly, svMP05-v15 corresponding to P-I type MP showed higher 
expression (Fragments Per Kilobase of exon per Million mapped fragments (FPKM) 2769.20) compare with svMP05-v1 corresponding to P-III type MP, HR1a (FPKM 247.38) (Figure 6D).

A. SVMP03_VMP-III-like gene
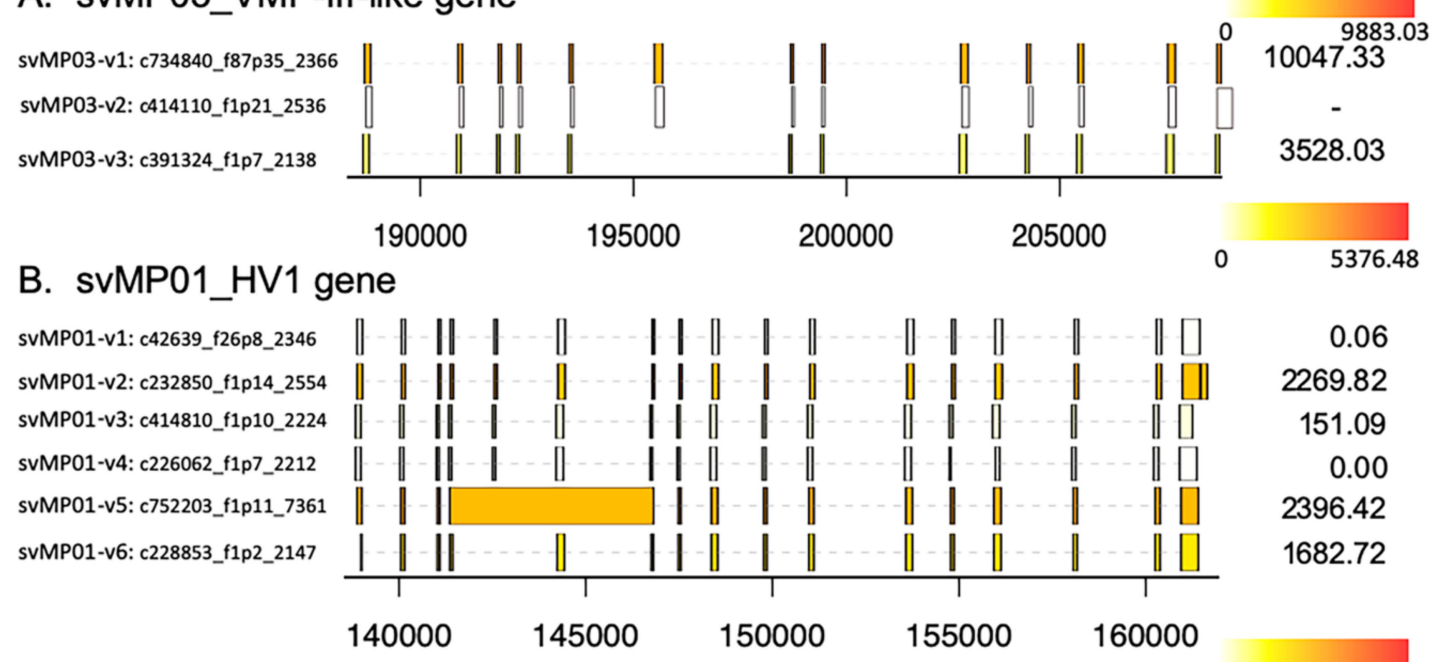

\section{C. svMP02_Flavorase gene}

SVMP02-v1: c42674_f12p2_2354
sVMP02-v2: c222143_f4p19_2794
sVMP02-v3: c250326_f1p0_3778
sVMP02-v4: c222615_f5p4_2265
SVMP02-v5: c256167_f6p4_2038
SVMP02-v6: c258735_f1p9_2479
SVMP02-v7: c410155_f1p8_5215

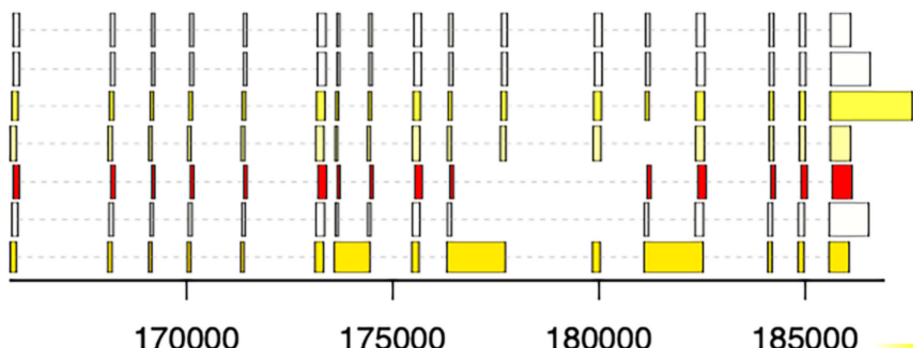

0

0.06

2269.82

151.09

0.00

2396.42

1682.72

\section{0}

175000

180000

D. sVMP05_HR1a-related gene

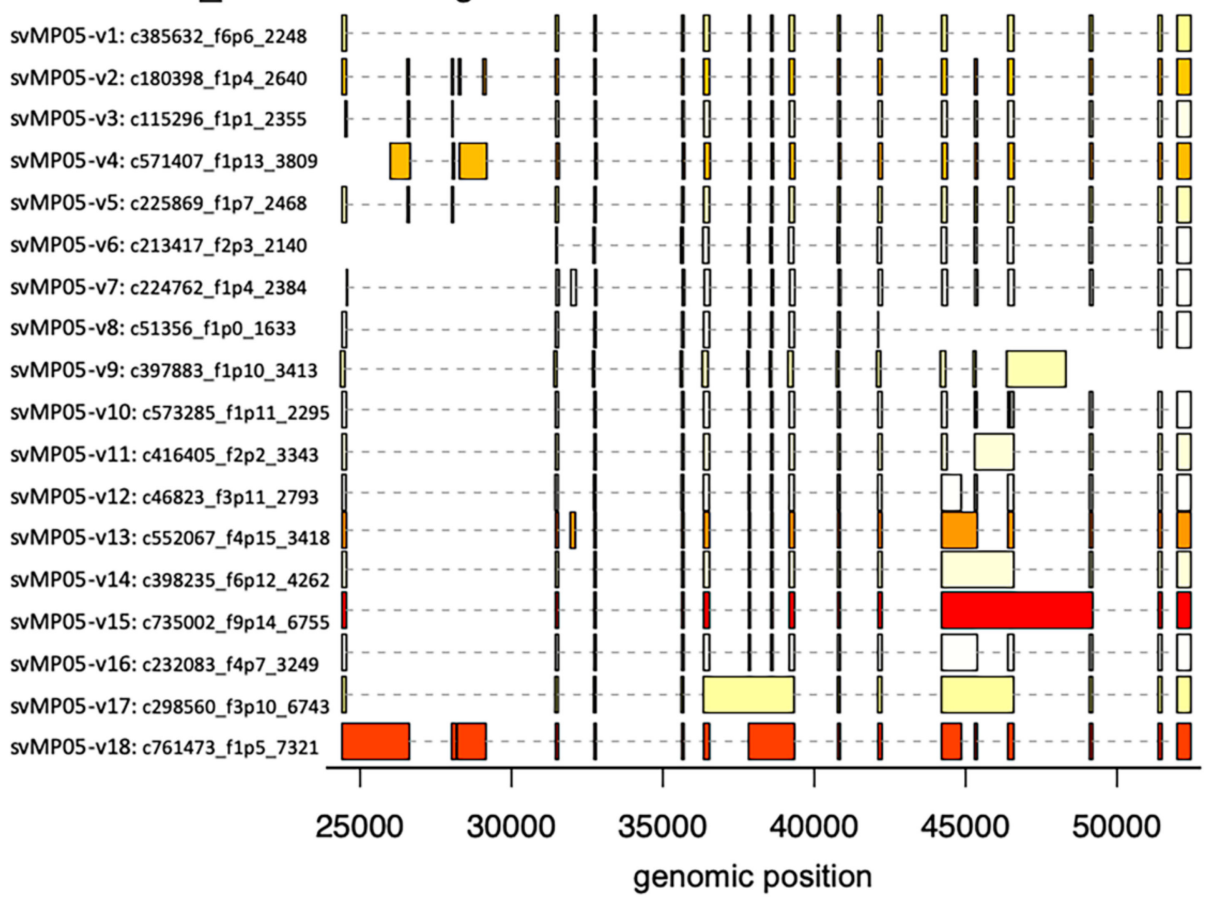

0

7549.91

747.96

3065.73

164.76

3384.06

481.32

0.00

0.00

0.00

514.42

0.00

278.66

0.00

4181.79

282.79

8193.75

0.00

692.53

6094.67

Figure 6. Cont. 


\section{E. svMP11_NaMP gene}

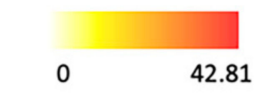

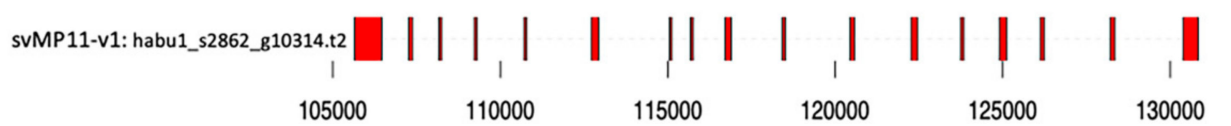

\section{F. svMP10_elegantin-like gene}

43.08

SVMP10-v1: c16287_f13p13_2020

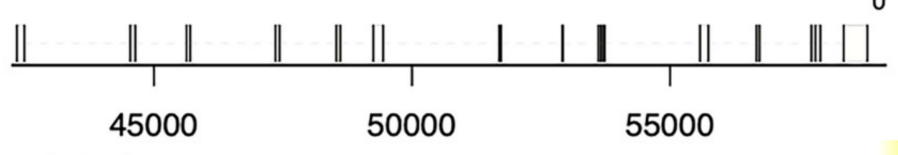

sVMP10-V1: c16287_t13p13_2020

45000

50000

55000

\section{G. sVMP07_HR2a-related gene}

$0 \quad 26683.6$

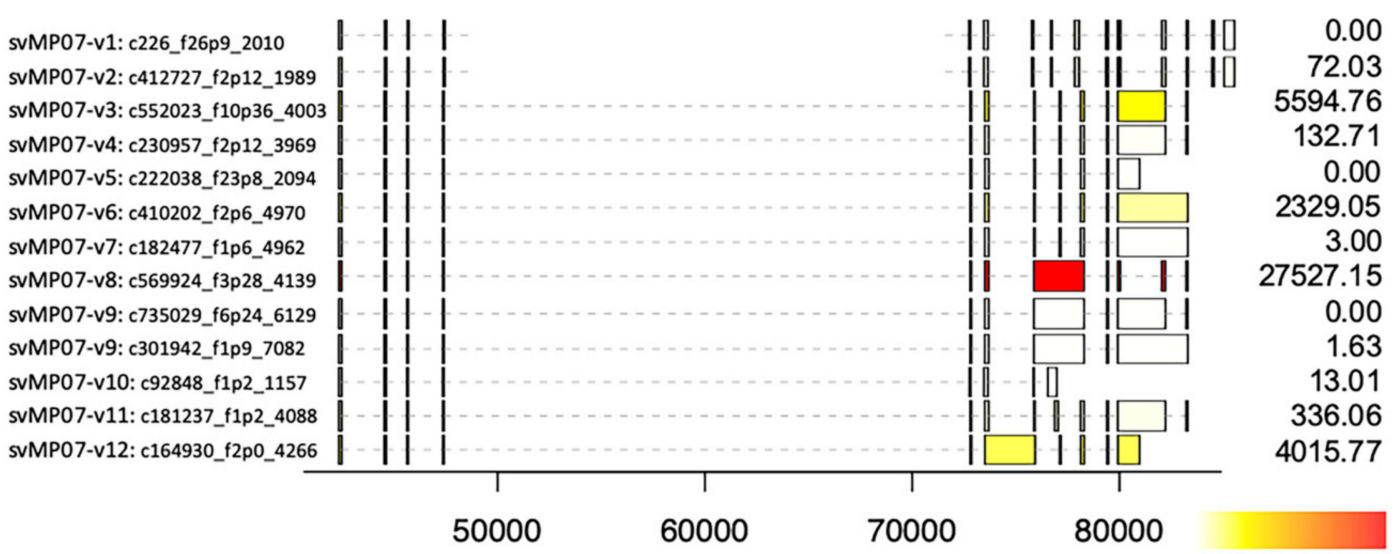

H. svMP08_flavoviridin gene

$0 \quad 62515.27$

SVMP08-v1: c222_f46p26_1997 SVMP08-V2: c734849_f1820p63_1998 SVMP08-V3: c222359_f4p23_2219 sVMP08-v4: c222126_f12p24_3060 SVMP08-v5: c9689_f1p6_1905 SVMP08-v6: c359165_f2p11_1737 SVMP08-v7: c80731_f1p5_1484 SVMP08-v8: c557711_f1p8_1601 SVMP08-v9: c222549_f3p4_2038 SVMP08-v10: c222077_f17p8_3057 SVMP08-v11: c552171_f2p7_3985 sVMP08-v12: c740798_f1p7_5424 SVMP08-v13: c576234_f1p8_3948 SVMP08-v14: c417811_f8p12_4120 SVMP08-v15: c589782_f1p11_3503 sVMP08-v16: c394718_f2p10_2465 sVMP08-v17: c585605_f1p3_3986
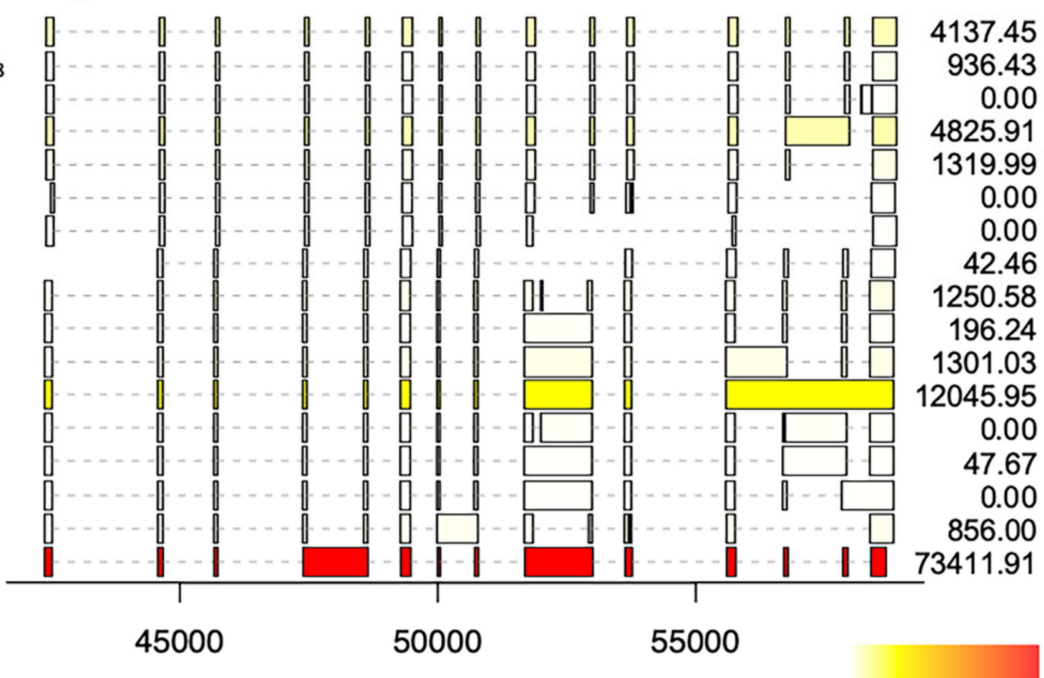

I. svMP06_H2 protease gene

SVMP06-V1: c225249_f1p9_1815 sVMP06-V2: c164899_f9p15_3671

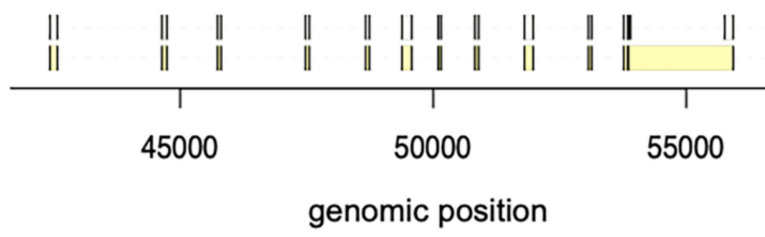

Figure 6. Structure and expression levels of distinct transvariant forms of svMPs genes in habu venom glands. (A) scMP03_VMP-III like, (B) svMP01_HV1, (C) svMP02_flavorase, (D) svMP05_HR1a, (E) svMP11_NaMP, (F) svMP10_elegantin-like, (G) svMP07_HR2a, (H) svMP08_flavoviridin, and (I) svMP06_H2 protease. Expression levels are shown on the right with FPKM values. 
As described, svMPs are classified into four different groups (P-I to P-IV). Different types of MP proteins may be produced from single MP genes such as svMP09-HR1b-related and svMP05-HR1a-related not only by proteolytic processing but also alternatively splicing, resulting in a variety of svMPs and disintegrin peptides.

2.3. Alternative Splicing of svSP Genes Produces mRNA Variants with Different Lengths of $3^{\prime} U T R$ or Inactive Short Forms Lacking C-Terminal Region

SPs are another major group of protein components of snake venom. We identified 11 habu svSP genes (svSP01 to svSP11) from the gene model of HabAm1 (Figure 2; Supplementary Table S2) [7]. svSP01 is known as flavoxobin, TLf1, or habutobin, which is the most abundantly expressed svSP in the venom gland [12-14]. It encodes a thrombin-like enzyme of 242 amino acids that specifically releases fibrinopeptide A from fibrinogen [12]. svSP02 corresponds to another major svSP transcript encoding a basic thrombin-like enzyme, TLf2. Comparative analyses of nucleotide sequences of $P$. flavoviridis svSPs with orthologues previously reported from other snake species [15] enabled us to assign SvSP03, svSP04, svSP05, and svSP09 genes to basic thrombin-like enzyme TLf3, a new SP homologous to stejnefibrase-1, flavorase, and an $\alpha$-fibrinogenase, respectively.

All svSP genes are composed of six exons, except svSP06 which has two new exons (Figures 2 and 7). A molecular phylogenetic tree of the $11 \mathrm{svSP}$ genes suggests that $s v S P 08$ is likely to be the earliest-diverged variant from the ancestral state, followed by multiple stages of duplications and divergence (Figure 2). As shown in Figures 2 and 7, in all 11 svSP genes, irrespective of the number of exons, the first exons correspond to $5^{\prime}$ UTRs. All of these svSPs share the same exon-intron junctions as the coding regions of mammalian kallikrein $(K L K)$ genes [16]. Together with the similarity of enzymatic activities and the conservation of primary and tertiary structures of SPs, it has been thought that the ancestral copy of all svSPs is a kallikrein-like SP rather than other types of SPs such as elastase. This further suggests that $s v S P 06$ gained two new exons, exons 7 and 8, resulting in extension of the $3^{\prime}$ UTR (Figure 2I).

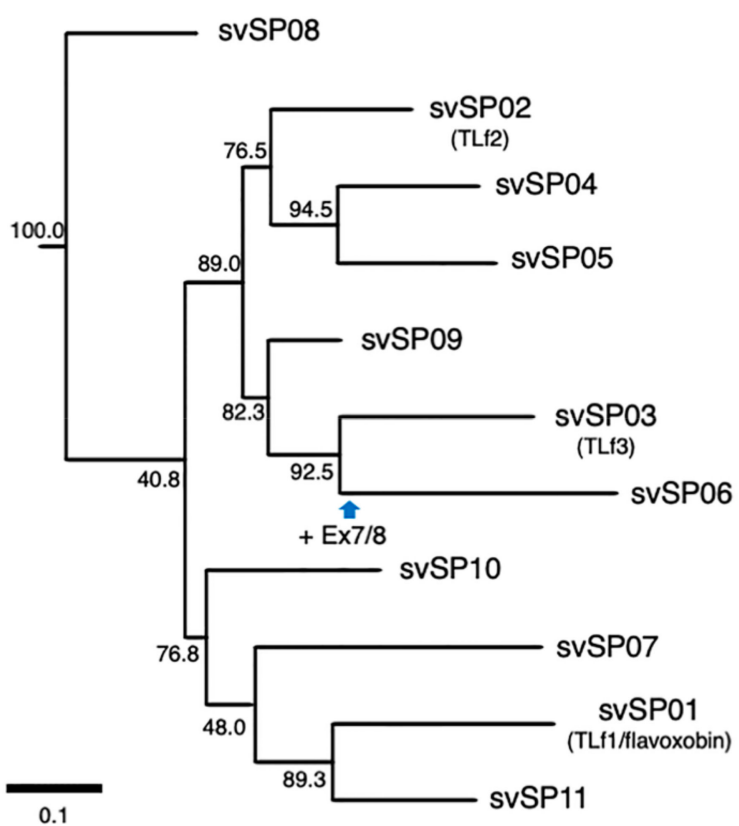

\section{Number of exons Transcripts}

$\begin{array}{ll}6 & 3 \\ 6 & 6 \\ 6 & 8 \\ 6 & 6 \\ 6 & 3 \\ 6 & 6 \\ 8 & 7 \\ 6 & 3 \\ 6 & 9 \\ 6 & 7 \\ 6 & 3\end{array}$

Figure 7. Molecular phylogeny of serine protease genes expressed in habu venom glands. A non-venom SP found in the habu genome, nvSP05-kallikrein, was used as an outgroup for rooting the tree. Bootstrap probabilities of 1000 replications are shown at each node. Deduced timing of gain of exons is shown by a blue arrow. 
A great variety of transcripts of $s v S P$ genes was observed, indicating extensive alternative splicing of $s v S P$ genes as seen in $s v M P$ genes (Figures 2 and 7). svSP08, svSP09, svSP10 and svSP11 express three transcript variants each, including the "original" transcript deduced from the gene model. svSP02-TLf2, svSP03-TLf3, and svSP05 are transcribed into six variants. svSP01-TLf1-flavoxobin and svSP06 are transcribed into seven variants. svSP04 is transcribed into eight variants. svSP07 is transcribed into nine variants. As seen in MP genes, we noticed several mechanisms to produce transcript variants. For example, among the eight transcripts expressed from svSP04, skipping of exon 2 was observed in five transcript variants, resulting in gain of a new start-codon in exon 3 of the original construct (svSP07-v3, svSP07-v4, svSP07-v5, svSP07-v6, svSP07-v7) (Figure 2E). Furthermore, variant transcripts completely or partially retaining intron 4 result in gaining a new stop-codon in the original intron 4 (svSP07-v5, svSP07-v6, svSP07-v7, and svSP07-v8). In addition, unlike svMP genes, the svSP transcript variants show variable lengths in the $3^{\prime}$-region of exon 6 due to multiple polyA signals (Figure 2A-D,G,H,K). Furthermore, we observed variant transcripts derived from trans-splicing involving exons in two to three tandemly arranged neighboring genes (Figure 8). For example, c252215_f1p2_2614 was transcribed from exons 1-5 of svSP03-TLf3 and exon 6 of svSP01-TLf1-flavoxobin which is located downstream of svSP03-TLf3 (Figure 8A). c258868_f2p6_2221 was transcribed from exons 1-3 of svSP01-TLf1-flavoxobin and exons 4-6 of svSP09 which is located downstream of svSP01-TLf1-flavoxobin (Figure 8A). c50972_f1p2_1888 was transcribed from exons 1-3 of svSP04, exons 4 and 5 of svSP05 and exon 6 of svSP08. c552059_f9p9_1588 was transcribed from exons 1-3 of svSPO4 and exons 4 and 5 of svSP05 (Figure 8B). c8598_f5p23_1588 was transcribed from exons 1 and 2 of svSP04 and exons 3-6 of svSP05 s (Figure 8B). c39314_f4p1_3574 was transcribed from exons 1-3 of svSP04 and exons 4-6 of svSP08 genes (Figure 8B). All of these trans-spliced products were supported by multiple Iso-Seq reads. Therefore, intrinsic trans-splicing serves as a previously unknown mechanism to enhance the diversity of final protein products of $s v S P$ genes.

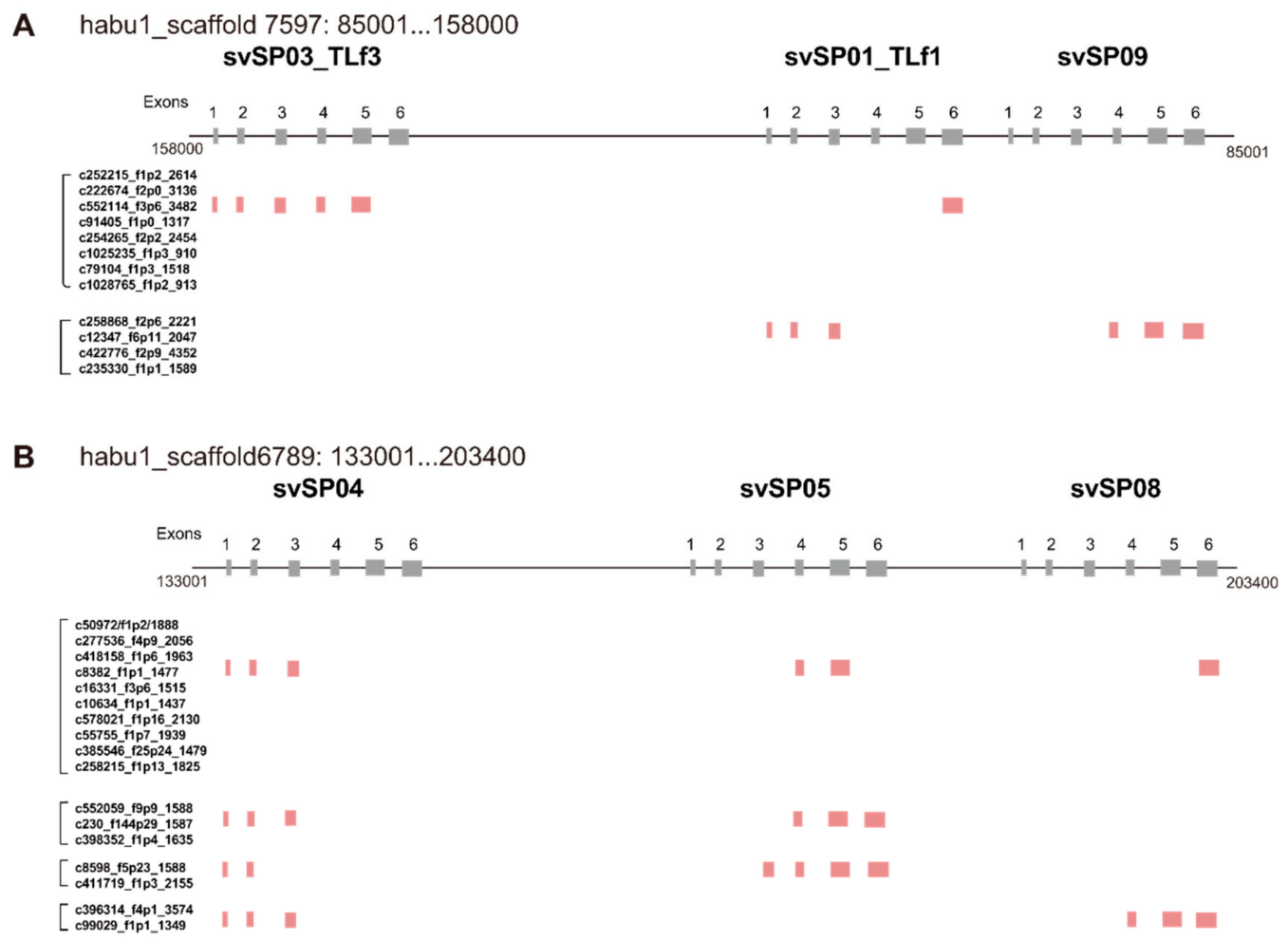

Figure 8. Chimeric transcripts generated by trans-splicing of clustered genes. Trans-spliced transcripts were identified from two regions of SP gene clusters, habu1_scaffold 7597: 85001...158000 (A) and habu1_scaffold6789: 133001...203400 (B). All of these trans-spliced transcripts were supported by multiple Iso-Seq reads. 


\subsection{VEGF Gains Its C-Terminal Modification by Alternative Splicing}

VEGFs are key regulators of vascular development during embryogenesis (vasculogenesis), blood-vessel formation (angiogenesis), skeletal growth, and reproductive functions [17]. We identified three VEGF genes from the gene model of HabAm1. One is the toxic copy, svVEGF01 (habu1_s565_g02679) encoding svVEGF-F, while the other two are non-toxic copies, nvVEGF01 (nv: non-venom; habu1_s6836_g17529) and nvVEGF02 (habu1_s9381_g19343) encoding VEGF-A and VEGF-C, respectively. In the current analysis we identified eight transcript variants expressed from svVEGF01 (Figure 3). The "original" transcript, svVEGF-v1 was the major transcript encoding the full-length length amino acid sequence exactly matching the one previously reported [18]. In contrast, all transcript variants carry variably shortened coding sequences. svVEGF-v2 gained a stop-codon in the retained intron 5. In spite of the difference in structures of exons 5 and 6, svVEGF-v3, svVEGF-v4, svVEGF-v5, and svVEGF-v6 commonly gained a stop-codon in the retained intron 3, resulting in an identical protein product. svVEGF-v7 gained a stop-codon in the retained intron 1, resulting in a highly truncated protein. svVEGF-v8 gained a new stop-codon in exon 3 due to skipping of exon 2, resulting in a very short protein.

\section{5. $P L A_{2}$ S Show No Alternative Splicing}

$\mathrm{PLA}_{2}$ (EC 3.1.1.4) catalyzes the hydrolysis of 2-acyl ester bonds of 3-sn-phosphoglycerides in the presence of $\mathrm{Ca}^{2+}$, to liberate 3-sn-lysophosphoglycerides and fatty acids. Habu snake genome contains nine types of group II venom PLA $A_{2}$ genes (svPLA $A_{2} 01$ to $s v P L A_{2} 09$ ) (Figure 4) [7], including a hemolytic [Asp49]PLA (svPLA $\left._{2} 01\right)$, edema-inducing basic [Asp49]PLA ${ }_{2}$ including PLX', PL-Y and PL-B (svPLA $\left.A_{2} 02\right)$, a weak neurotoxin PLA-N (svPLA $\left.A_{2} 03\right)$, and [Lys49]PLA ${ }_{2}$ myotoxins ( $s v P L A_{2} 04$ to svPLA $A_{2} 06$ corresponding to BPI, BPII, and BPIII, respectively), were expressed in the venom gland with high number of transcripts (16-3206) compared with three genes, svMP, svSPS, and svVEGF (Figure 4). From the structure of each transcript, svPLA ${ }_{2} \mathrm{~s}$ have no alternative splicing although these $P L A_{2}$ genes consist of four exons (Figure 4).

Total number of transcripts for $\mathrm{PLA}_{2} \mathrm{~s}$ was estimated to be 9654 , ten percent of 97,405 Iso-Seq reads in venom gland. Compared with $\mathrm{PLA}_{2} \mathrm{~s}$, total number of reads for svMP, svSPs, and svVEGF were low with 1777, 476, and 20, respectively. On the other hand, FPKM values of svPLA 2 s, svMPs, svSPs, and svVEGF in venom glands from two individuals (VG1/VG2) were estimated to be 3590.15/13728.43, 6140.1/1138.4, 149.2/630.8, and 90.4/116.9, respectively. These results suggest that the expression levels of svPLA ${ }_{2}$, svMPs, svSPs, and svVEGF estimated by two different methods, PacBio Iso-Seq reads and Illumina RNA-seq, were almost parallel. However, the number of full-length transcripts (Iso-Seq reads) for svMPs were very low, more than the difference of expression levels probably due to their short reads and the existence of unidentified svMP genes. Furthermore, PLA2s are much easier to identify (only four exons, $<450$ bp usually for the coding sequence) so the models may be much better for them. With svMPs having 15-17 exons ( $>1400$ for Type 2 and $>1800$ for Type 3), there is a lot more possibility of errors in annotation.

\section{Discussion}

Previous studies have suggested the occurrence of alternative splicing of venomic SP genes in Vipera lebetina and Bitis gabonica rhinoceros $[9,10]$. Recent high-throughput transcriptomics (RNA-seq) demonstrated a highly divergent profile of mRNA species encoded by venom genes, and the involvement of alternative splicing is suggested to contribute to transcriptome variation [4-6]. Genome-wide transcriptome analyses, including alternative-splicing variants of venom genes, have not been previously reported. By genome-based comprehensive analysis of the full-length transcript information provided by Iso-Seq reads of the venom gland in P. flavoviridis, we showed that only three of the 18 families, namely $s v M P$, svSP, and $s v V E G F$, produce highly diverse transcript variants by alternative-splicing in venom glands. Thus, the diversity of venom transcripts in $P$. flavoviridis is 
provided by two distinct mechanisms; namely, alternative splicing, as we reported in the current study, and the increment of copy number followed by accelerated evolution, as we reported earlier [7].

The present study disclosed "double-decker" mechanisms; namely, extensive gene duplication followed by the rearrangements of exon/intron structures. For examples, in the case of the 24 copies of svMP genes, five copies preserved the original arrangement consisting of 17 exons, whereas four copies are the "deleted" construct consisting of 15 exons, of which the terminal one is "further deleted" carrying only 13 exons. The most extensive variation by alternative splicing was noticed in svMPs; that is, transcript variants involving exon skipping or intron retention (fused exons) incorporated a new stop-codon, resulting in the production of diverse variants corresponding to different types of MPs. For example, from the genes encoding P-III-type MPs, HR1a, and HR1b, all three types of variants were produced, P-I, P-II, and P-III. While from the P-II-type MP genes such as svMP04-jerdonitin-like, svMP07-HR2a-related, and svMP08-flavoridin, two types variants were produced, P-I and P-II. These transcript variants for each MP were confirmed by HISAT, StringTie, and Ballgown with Illumina RNA-Seq data (Figure 6). The diversification mechanism by alternative splicing may be advantageous for svMPs because they are mosaic proteins belonging to a disintegrin and metalloprotease (ADAM) family, which contains the disintegrin and Cys-rich domains encoded by separate exons, in addition to MP and pre-prosequence domains (Figure 5A).

In the case of the 11 copies of $s v S P$ genes, 10 copies retained the original architecture consisting of six exons, whereas one copy gained two new exons, resulting in eight exons. Many of these rearrangements involve gain and loss of start- and stop-codons. We identified transcript variants for all of the $11 \mathrm{svSP}$ genes carrying intron retentions as well as exon skipping, resulting in gains of premature stop-codons or of secondary start-codons. Some transcript variants such as c768983_f1p7_7228 of svSP01-TLf1-flavoxobin and c735101_f3p4_5942 of svSP02-TLf2 are very small and naturally devoid of catalytic activity as proteinases due to lack of the catalytic residue, Ser195. Our unpublished data show that TLf2 enhances the myonecrotic activity of [Lys49]PLA 2 , although TLf2 was hydrolytically inactive due to replacement of its catalytic residue His57 with Arg (Ogawa et al., in preparation). This indicates the possible functions of svSP fragmentary products derived from short-transcript variants because TLf2 exhibits a unique activity independent of its protease activity.

In addition to the two major protein components in the venom MP and SP, alternative splicing also serves to enhance the diversity in transcripts expressed from svVEGF (Figure 3). Transcript variants of svVEGF carry variable stop-codon positions, such as retained intron 1 , exon 3 , retained intron 3 , and retained intron 5 and exon 6 (original). VEGFs in snake venoms are known to show highly strict specificities of receptor-binding compared to mammalian VEGFs. For example, vammin and VR-1, isolated from the venoms of Vipera ammodytes and Daboia russelii, respectively, bind only kinase insert domain receptor (KDR) with high affinity but not to other VEGF receptors [19]. It is known that the C-terminal regions of VEGFs are one of the determinants for receptor specificities [20,21]. Yamazaki et al. [18] has also reported that svVEGF of $P$. flavoviridis preferentially binds to Flt-1 rather than to KDR, unlike vammin and VR-1 [22]. Thus, transcript variants of svVEGFs with variable C-terminal regions are likely to show differences in receptor specificities, rendering additional diversity in physiological functions of the venom.

Besides the normal cis-spliced transcripts discussed above, we also observed trans-spliced transcript variants of $s v S P$ s expressed from genes tandemly clustered so that the pre-mRNA spanning multiple genes is able to be expressed (Figure 8). This may serve as yet another mechanism, which is previously unknown, to enhance the diversity of venom protein products. In the current study, we reported trans-splicing involving two regions of clustered genes, namely svSP03-svSP01-svSP09 and svSP04-svSP05-svSP08. In our previous study, however, we identified more cases of gene clusters of MPs, $S P S$, and CTLPS in the habu genome. Therefore, it is highly likely that trans-splicing also contributes to the expression of other neighboring genes located in the gene cluster, which needs to be elucidated in future study. 
Because recent transcriptomic and proteomic analyses of snake venoms have revealed that the venom components often show variations within as well as between species, it is considered that diversification of these venomous proteins at the transcriptional level is advantageous for physiological and environmental changes, including prey species [23-27]. These venom variations may be correlated with geographic location, diverse environments, sexual dimorphism, and feeding behaviors. In the current study, we revealed multilayered mechanisms that enhance the diversity of venom protein products, namely, alternative splicing, and trans-splicing. These are likely to serve as intrinsic mechanisms to render physiological and adaptive changes to the protein components of snake venoms.

\section{Conclusions}

In the present study, we found that alternative splicing was observed in three venom protein gene families, $M P, S P$, and $V E G F$, that is, a total of 81,61 , and 8 transcript variants, respectively. Especially, individual genes of $M P S$ are transcribed into different classes of MPs by alternative splicing. We also observed trans-splicing among the clustered SP genes. No other venom genes such as PLA2s exhibited alternative splicing. Thus, our results indicate a significant contribution of mRNA alternative and trans-splicing in the production of highly variable transcripts of venom genes in the habu snake.

\section{Materials and Methods}

\subsection{Biological Materials}

P. flavoviridis individuals were collected from Amami-Oshima Island, Kagoshima, Japan in 2011. One female individual was used for RNA extraction from venom gland. Crude venom was collected from adult $P$. flavoviridis from Amami-Oshima, Japan, and lyophilized. It was stored at $-30^{\circ} \mathrm{C}$ until use.

\subsection{Transcriptome Analyses by PacBio Reads}

Transcriptome analyses by PacBio reads were conducted as described in the previous our work [7]. Briefly, cDNA libraries from the venom gland for PacBio sequencing were prepared by using the manufacturer's protocol with a SMARTer Pico PCR cDNA Synthesis Kit (TAKARA Clontech) and SMRTbell Template Preparation Kit 1.0 (PacBio). Longer cDNAs were enriched with a SageELF system (Sage Science, Inc.). Sequencing was performed on a PacBio RS II, yielding a total of 179,143,509 bps of $2300 \mathrm{bp}$ average read length. N50 of PacBio reads was 5000 and not required for further assembly. Most of these reads are long enough to be full-length transcripts and directly annotated with BLASTX against UniProt. PacBio reads are publicly available from DDBJ under accession no. DRA006601.

\subsection{Transcriptome Analyses by Illumina}

For transcriptome analyses by Illumina RNA-Seq, two individual venom glands (VG1 and VG2) were used. Total RNA was extracted by using a standard TRIzol protocol procedure (Thermo Fisher Scientific), and cDNA libraries were prepared using an NEBNext ${ }^{\circledR}$ Ultra ${ }^{\mathrm{TM}}$ Directional RNA Library Prep Kit for Illumina (New England Biolabs). RNA quality was checked with an Agilent Technologies 2100 Bioanalyzer using an Agilent RNA 6000 Nano Kit. Sequencing was performed using an Illumina Hiseq2500. The sequencing quality was checked by FastQC [28], and low complexity sequences were removed by PRINSEQ [29]. The raw Illumina reads were quality filtered (Q20) and trimmed out $5-10 \mathrm{bp}$ on both ends of the reads to remove bias and low-quality bases by using Trimmomatic [30]. De novo assembly of whole RNA sequence reads was performed using a de Bruijn graph-based program, Trinity [31]. Assembled transcripts were annotated with BLASTX against UniProt. Illumina reads are publicly available from DDBJ under accession no. DRA006600.

\subsection{Mapping of Transcriptome Data on Genome Sequence and Detection of Transcript Variants}

All PacBio Iso-Seq transcript reads were mapped against the genome assembly, HabAm1 using BLAT [32] and PASA [33]. HabuAm1, the genome sequence data of P. flavoviridis previously reported [7], 
can be accessed in BioSamples SAMD00115727 (DRX006596-DRA006599). Accession numbers for scaffolds are BFFQ01000001-BFFQ01084502 (84,502 entries).

PacBio Iso-Seq transcript data encoding venom proteins was checked and corrected via a habu genome browser (Supplementary Figure S1). PacBio Iso-Seq transcript data for venom proteins was also analyzed by blast search on habu1_venom pac bio_prot and habu1_venom_pac_bio_nucl database of the habu genome browser using amino acid sequence and nucleotide sequence of each transcript variant as query sequence. Finally, we confirmed the data by multiple alignments of transcripts against each venom gene with ClustalW [34] and manual curation by comparing with genome sequence to remove the sequencing errors and/or allelic variation. Short Iso-Seq reads without a start Met codon and/or polyA signal were also omitted from data. Then, the number of validated transcript variants were counted.

\subsection{Bioinfomatic analysis}

A genome browser was prepared using the assembled genome sequences using the JavaScript-based Genome Browser (JBrowse) 1.11.6 [35]. The assembled sequence and gene models can be found at http://marinegenomics.oist.jp/habu/.

Alignment of amino acid and nucleotide sequences of each protein family was first performed using ClustalW online at http://clustalw.ddbj.nig.ac.jp. Then the alignment of nucleotide sequences was made based upon the amino acid sequence alignment. We carefully and repeatedly confirmed the correspondence between nucleotide sequences of the genome and mRNAs and amino acid sequences of the predicted proteins.

Phylogenetic trees were constructed by the maximum-likelihood method using IQ-TREE (http: //www.iqtree.org) [36], based on aligned nucleotide sequences. Numbers on branches are bootstrap values with $1000 \times$ resampling. The optimal evolutionary model for each phylogenetic tree was selected using ModelFinder [37] implemented in IQ-TREE.

The expression level of transcript variants in venom gland was analyzed by Pertea's protocols using open source software tools [38], HISAT (hierarchical indexing for spliced alignment of transcripts. Ver. 2.1.0) [39], StringTie (Ver. 1.3.4d) [40], and Ballgown (Ver. 2.16.0) [41] with Illumina RNA-Seq data.

Supplementary Materials: The following are available online at http://www.mdpi.com/2072-6651/11/10/581/s1, Figure S1: Habu genome browser, Table S1: Metalloproteinase (MP) genes and representative transcripts expressed in the habu venom gland, Table S2: Serine protease (SP) genes and representative transcripts expressed in the habu venom gland.

Author Contributions: T.O. and H.S. designed experiments and performed analyses. T.O., N.O.-U., T.C., H.N., S.H., Y.F., M.O., and H.S. prepared materials from live specimens. H.S. and S.Y. performed RNA sequencing. T.O., K.H., A.I., H.Y., N.S., and H.S. performed bioinformatics analyses. T.O., N.O.-U., N.S., and H.S. supervised the project and wrote the manuscript with contributions from other all authors.

Funding: This work was supported by Grants-in-Aid of MEXT, Japan (\#25440214 and \#18H02498 to H.S., \#24651130 and \#23107505 to T.O.), and by an Internal Fund to the Marine Genomics Unit, Okinawa Institute of Science and Technology Graduate University (OIST). This work was partly supported by the Cooperative Research Project Program of the Medical Institute of Bioregulation, Kyushu University (T.O., N.O.-U.).

Acknowledgments: We thank members of the Marine Genomics and DNA sequencing sections of OIST. Supercomputing resources were provided by the Human Genome Center, the University of Tokyo (http: //sc.hgc.jp/shirokane.html), and the National Institute of Genetics (https://sc2.ddbj.nig.ac.jp/index.php/en/).

Conflicts of Interest: The authors declare no conflict of interest. 


\section{References}

1. Fry, B.G.; Vidal, N.; Norman, J.A.; Vonk, F.J.; Scheib, H.; Ramjan, S.F.R.; Kuruppu, S.; Fung, K.; Hedges, S.B.; Richardson, M.K.; et al. Early evolution of the venom system in lizards and snakes. Nature 2006, 439, 584-588. [CrossRef]

2. Fry, B.G.; Vidal, N.; Van Der Weerd, L.; Kochva, E.; Renjifo, C. Evolution and diversification of the Toxicofera reptile venom system. J. Proteom. 2009, 72, 127-136. [CrossRef] [PubMed]

3. Ikeda, N.; Chijiwa, T.; Matsubara, K.; Oda-Ueda, N.; Hattori, S.; Matsuda, Y.; Ohno, M. Unique structural characteristics and evolution of a cluster of venom phospholipase A2 isozyme genes of Protobothrops flavoviridis snake. Gene 2010, 461, 15-25. [CrossRef]

4. Castoe, T.A.; Hall, K.T.; Mboulas, M.L.G.; Gu, W.; De Koning, A.J.; Fox, S.E.; Poole, A.W.; Vemulapalli, V.; Daza, J.M.; Mockler, T.; et al. Discovery of highly divergent repeat landscapes in snake genomes using high-throughput sequencing. Genome Biol. Evol. 2011, 3, 641-653. [CrossRef]

5. Aird, S.D.; Watanabe, Y.; Villar-Briones, A.; Roy, M.C.; Terada, K.; Mikheyev, A.S. Quantitative high-throughput profiling of snake venom gland transcriptomes and proteomes (Ovophis okinavensis and Protobothrops flavoviridis). BMC Genom. 2013, 14, 790. [CrossRef] [PubMed]

6. Aird, S.D.; Aggarwal, S.; Villar-Briones, A.; Tin, M.M.Y.; Terada, K.; Mikheyev, A.S. Snake venoms are integrated systems, but abundant venom proteins evolve more rapidly. BMC Genom. 2015, 16, 647. [CrossRef] [PubMed]

7. Shibata, H.; Chijiwa, T.; Oda-Ueda, N.; Nakamura, H.; Yamaguchi, K.; Hattori, S.; Matsubara, K.; Matsuda, Y.; Yamashita, A.; Isomoto, A.; et al. The habu genome reveals accelerated evolution of venom protein genes. Sci. Rep. 2018, 8, 11300. [CrossRef] [PubMed]

8. Cousin, X.; Bon, S.; Massoulie', J.; Bon, C. Identification of a Novel Type of Alternatively Spliced Exon from the Acetylcholinesterase Gene of Bungarus fasciatus. J. Biol. Chem. 1998, 273, 9812-9820. [CrossRef]

9. Siigur, E.; Aaspo llu, A.; Siigur, J. Sequence diversity of Vipera lebetina snake venom gland serine proteinase homologs-result of alternative-splicing or genome alteration. Gene 2001, 263, 199-203. [CrossRef]

10. Vaiyapuri, S.; Wagstaff, S.C.; Harrison, R.A.; Gibbins, J.M.; Hutchinson, E.G. Evolutionary Analysis of Novel Serine Proteases in the Venom Gland Transcriptome of Bitis gabonica rhinoceros. PLoS ONE 2011, 6, e21532. [CrossRef]

11. Fox, J.W.; Serrano, S.M. Structural considerations of the snake venom metalloproteinases, key members of the M12 reprolysin family of metalloproteinases. Toxicon 2005, 45, 969-985. [CrossRef] [PubMed]

12. Shieh, T.C.; Tanaka, S.; Kihara, H.; Ohno, M.; Makisumi, S. Purification and Characterization of a Coagulant Enzyme from Trimeresurus flavoviridis Venom. J. Biochem. 1985, 98, 713-721. [CrossRef]

13. Deshimaru, M.; Ogawa, T.; Nakashima, K.I.; Nobuhisa, I.; Chijiwa, T.; Shimohigashi, Y.; Fukumaki, Y.; Niwa, M.; Yamashina, I.; Hattori, S.; et al. Accelerated evolution of crotalinae snake venom gland serine proteases. FEBS Lett. 1996, 397, 83-88. [CrossRef]

14. Sunagawa, M.; Nakamura, M.; Kosugi, T. Cloning of habutobin cDNA and antithrombotic activity of recombinant protein. Biochem. Biophys. Res. Commun. 2007, 362, 899-904. [CrossRef] [PubMed]

15. Serrano, S.M.T.; Hagiwara, Y.; Murayama, N.; Higuchi, S.; Mentele, R.; Sampaio, C.A.M.; Camargo, A.C.M.; Fink, E. Purification and characterization of a kinin-releasing and fibrinogen-clotting serine proteinase (KN-BJ) from the venom of Bothrops jararaca, and molecular cloning and sequence analysis of its cDNA. JBIC J. Biol. Inorg. Chem. 1998, 251, 845-853. [CrossRef] [PubMed]

16. Van Leeuwen, B.H.; Evans, B.A.; Tregear, G.W.; Richards, R.I. Mouse glandular kallikrein genes. Identification, structure, and expression of the renal kallikrein gene. J. Biol. Chem. 1986, 261, 5529-5535.

17. Ferrara, N.; Gerber, H.P.; LeCouter, J. The biology of VEGF and its receptors. Nat. Med. 2003, 9, 669-676. [CrossRef]

18. Yamazaki, Y.; Matsunaga, Y.; Tokunaga, Y.; Obayashi, S.; Saito, M.; Morita, T. Snake Venom Vascular Endothelial Growth Factors (VEGF-Fs) Exclusively Vary Their Structures and Functions among Species. J. Biol. Chem. 2009, 284, 9885-9891. [CrossRef]

19. Yamazaki, Y.; Takani, K.; Atoda, H.; Morita, T. Snake Venom Vascular Endothelial Growth Factors (VEGFs) Exhibit Potent Activity through Their Specific Recognition of KDR (VEGF Receptor 2). J. Biol. Chem. 2003, 278, 51985-51988. [CrossRef] 
20. Nieminen, T.; Toivanen, P.I.; Rintanen, N.; Heikura, T.; Jauhiainen, S.; Airenne, K.J.; Alitalo, K.; Marjomäki, V.; Ylä-Herttuala, S. The impact of the receptor binding profiles of the vascular endothelial growth factors on their angiogenic features. Biochim. Biophys. Acta BBA Gen. Subj. 2014, 1840, 454-463. [CrossRef]

21. Toivanen, P.I.; Nieminen, T.; Laakkonen, J.P.; Heikura, T.; Kaikkonen, M.U.; Ylä-Herttuala, S. Snake venom VEGF Vammin induces a highly efficient angiogenic response in skeletal muscle via VEGFR-2/NRP specific signaling. Sci. Rep. 2017, 7, 5525. [CrossRef] [PubMed]

22. Takahashi, H.; Hattori, S.; Iwamatsu, A.; Takizawa, H.; Shibuya, M. A novel snake venom vascular endothelial growth factor (VEGF) predominantly induces vascular permeability through preferential signaling via VEGF receptor-1. J. Biol. Chem. 2005, 279, 46304-46314. [CrossRef] [PubMed]

23. Alape-Girón, A.; Sanz, L.; Escolano, J.; Flores-Díaz, M.; Madrigal, M.; Sasa, M.; Calvete, J.J. Snake Venomics of the Lancehead Pitviper Bothrops asper: Geographic, Individual, and Ontogenetic Variations. J. Proteome Res. 2008, 7, 3556-3571. [CrossRef] [PubMed]

24. Núñez, V.; Cid, P.; Sanz, L.; De La Torre, P.; Angulo, Y.; Lomonte, B.; Gutiérrez, J.M.; Calvete, J.J. Snake venomics and antivenomics of Bothrops atrox venoms from Colombia and the Amazon regions of Brazil, Perú and Ecuador suggest the occurrence of geographic variation of venom phenotype by a trend towards paedomorphism. J. Proteom. 2009, 73, 57-78. [CrossRef] [PubMed]

25. Calvete, J.J.; Sanz, L.; Pérez, A.; Borges, A.; Vargas, A.M.; Lomonte, B.; Angulo, Y.; Gutiérrez, J.M.; Chalkidis, H.M.; Mourão, R.H.; et al. Snake population venomics and antivenomics of Bothrops atrox: Paedomorphism along its transamazonian dispersal and implications of geographic venom variability on snakebite management. J. Proteom. 2011, 74, 510-527. [CrossRef]

26. Augusto-De-Oliveira, C.; Stuginski, D.R.; Kitano, E.S.; Andrade-Silva, D.; Liberato, T.; Fukushima, I.; Serrano, S.M.T.; Zelanis, A. Dynamic Rearrangement in Snake Venom Gland Proteome: Insights into Bothrops jararaca Intraspecific Venom Variation. J. Proteome Res. 2016, 15, 3752-3762. [CrossRef]

27. Calvete, J.J. Venomics: Integrative venom proteomics and beyond. Biochem. J. 2017, 474, 611-634. [CrossRef]

28. Andrews, S. FastQC: A quality control tool for high throughput sequence data. 2010-2018 (ver.0.11.8). Available online: http://www.bioinformatics.babraham.ac.uk/projects/fastqc/ (accessed on 9 October 2019).

29. Schmieder, R.; Edwards, R. Quality control and preprocessing of metagenomic datasets. Bioinformatics 2011, 27, 863-864. [CrossRef]

30. Bolger, A.M.; Lohse, M.; Usadel, B. Trimmomatic: A flexible trimmer for Illumina sequence data. Bioinformatics 2014, 30, 2114-2120. [CrossRef]

31. Haas, B.J.; Papanicolaou, A.; Yassour, M.; Grabherr, M.; Blood, P.D.; Bowden, J.; Couger, M.B.; Eccles, D.; Li, B.; Lieber, M.; et al. De novo transcript sequence reconstruction from RNA-seq using the Trinity platform for reference generation and analysis. Nat. Protoc. 2013, 8, 1494-1512. [CrossRef]

32. Kent, W.J. BLAT-The BLAST-Like Alignment Tool. Genome Res. 2002, 12, 656-664. [CrossRef] [PubMed]

33. Haas, B.J.; Delcher, A.L.; Mount, S.M.; Wortman, J.R.; Smith, R.K.; Hannick, L.I.; Maiti, R.; Ronning, C.M.; Rusch, D.B.; Town, C.D.; et al. Improving the Arabidopsis genome annotation using maximal transcript alignment assemblies. Nucleic Acids Res. 2003, 31, 5654-5666. [CrossRef]

34. Larkin, M.; Blackshields, G.; Brown, N.; Chenna, R.; Mcgettigan, P.; Mc William, H.; Valentin, F.; Wallace, I.; Wilm, A.; López, R.; et al. Clustal W and Clustal X version 2.0. Bioinformatics 2007, 23, 2947-2948. [CrossRef] [PubMed]

35. Skinner, M.E.; Uzilov, A.V.; Stein, L.D.; Mungall, C.J.; Holmes, I.H. JBrowse: A next-generation genome browser. Genome Res. 2009, 19, 1630-1638. [CrossRef] [PubMed]

36. Nguyen, L.T.; Schmidt, H.A.; Von Haeseler, A.; Minh, B.Q. IQ-TREE: A fast and effective stochastic algorithm for estimating maximum likelihood phylogenies. Mol. Biol. Evol. 2015, 32, 268-274. [CrossRef] [PubMed]

37. Kalyaanamoorthy, S.; Minh, B.Q.; Wong, T.K.F.; Von Haeseler, A.; Jermiin, L.S. ModelFinder: Fast model selection for accurate phylogenetic estimates. Nat. Methods 2017, 14, 587-589. [CrossRef]

38. Perţea, M.; Kim, D.; Pertea, G.M.; Leek, J.T.; Salzberg, S.L. Transcript-level expression analysis of RNA-seq experiments with HISAT, StringTie and Ballgown. Nat. Protoc. 2016, 11, 1650-1667. [CrossRef]

39. Kim, D.; Langmead, B.; Salzberg, S.L. HISAT: A fast spliced aligner with low memory requirements. Nat. Methods 2015, 12, 357-360. [CrossRef]

40. Pertea, M.; Pertea, G.M.; Antonescu, C.M.; Chang, T.C.; Mendell, J.T.; Salzberg, S.L. Transcript-level expression analysis of RNA-seq experiments with HISAT, StringTie and Ballgown. Nat. Biotechnol. 2015, 33, $290-295$. [CrossRef] 
41. Fu, J.; Frazee, A.C.; Collado-Torres, L.; Jaffe, A.E.; Leek, J.T. Ballgown: Flexible, Isoform-Level Differential Expression Analysis. R Package Version 2.16.0. 2019. Available online: https://rdrr.io/bioc/ballgown/ (accessed on 9 October 2019). 